\title{
A boundary element method for steady infiltration from periodic channels
}

\section{Moh. Ivan Azis* David L.Clements ${ }^{\dagger} \quad$ Maria Lobo ${ }^{\ddagger}$}

(Received 1 June 2001; revised 30 October 2002)

\begin{abstract}
The matric flux potential and horizontal and vertical flux distributions are obtained for periodic irrigation channels by using boundary integral equation techniques. Numerical results are given for the special cases of semicircular and rectangular channels and the results compared with those of Batu [Soil Science Society of America Journal, 42:545549, 1978] and Warrick and Lomen [Soil Science Society of America Journal, 40:639-643, 1976] for a flat strip. The results show that the matric flux potential associated with the flat strip and semicircular channel are similar; whereas for the particular rectangular channel considered the matric flux potential is subtantially increased in the region adjacent to the channel.
\end{abstract}

*Department of Applied Mathematics, University of Adelaide, Australia.

${ }^{\dagger}$ as above, mailto:dclement@maths.adelaide.edu.au

$\ddagger$ as above, mailto:mlobo@maths.adelaide.edu.au

${ }^{0}$ See http://anziamj.austms.org.au/V44/CTAC2001/Azi1 for this article,

(C) Austral. Mathematical Soc. 2003. Published 1 April 2003. ISSN 1446-8735 


\section{Contents}

1 Introduction

C62

2 Statement of the problem

C63

3 Fundamental equations

C64

4 Boundary integral equation

C67

5 Numerical results

C68

6 Summary

C76

References

C77

\section{Introduction}

Philip [5, 6, 7], Wooding [11], Raats [8, 9], Batu [2], Zachmann and Thomas [12] have obtained solutions for steady infiltration from point, line and areal sources. Also Warrick [10], Lomen and Warrick [4] have obtained time-dependent solutions for point, line, strip and disc sources. For steady infiltration from a ditch Batu [2] has presented both theoretical and experimental results.

The present paper employs boundary integral equations to consider steady infiltration from periodic ditch sources (see Figure 1). Use of this method enables the matric flux potential associated with infiltration from periodic channels to be investigated for a variety of channel shapes which have not been considered by previous authors. The results obtained for some particular cases indicates how the method may be readily used to determine the influence of the channel shape on the matric flux potential in regions adjacent to the 


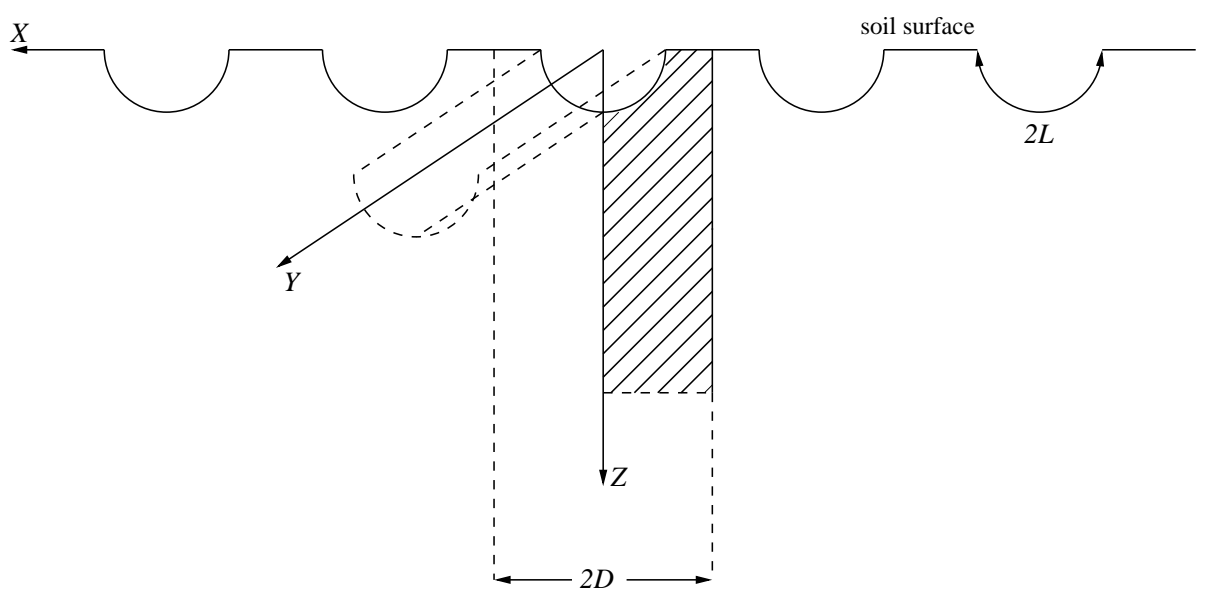

Figure 1: Periodic strip sources

channels. The analysis is based on a linearization, as proposed by Batu [1], of the flow equation in terms of the matric flux potential. The solutions are of interest in connection with furrow irrigation.

\section{Statement of the problem}

Referred to a Cartesian frame $O X Y Z$ consider an isotropic soil lying in the region $Z>0$ with $O Z$ vertically downwards. The boundary of the soil lies in the plane $Z=0$ except for a periodic series of channels which have a geometry which does not vary in the $O Y$ direction and further is such that it is symmetrical about the plane $X=0$ (see Figure 1). In this work, we investigate three different channel shapes. Specifically, the flat strip, semicircular and rectangular channels. The geometry of each channel is presented in Figures 2, 3 and 4. Each of the identical channels has surface area $2 L$ per unit length in the $O Z$-direction, where $L$ is a reference 
length. Also the distance between corresponding points on adjoining channels is denoted by $2 D$, where $D$ is a reference length. We assume that the water table is located at infinity and each of the channels is filled with water.

It is required to find the matric flux potential $\Theta(X, Z)$ throughout the soil $Z>0$.

\section{$3 \quad$ Fundamental equations}

The relationship between the hydraulic conductivity $K(h)$ (unit length per unit time) of unsaturated soil and the hydraulic conductivity $K_{s}$ of saturated soil is taken in the exponential form (see Batu [1])

$$
K(h)=K_{s} \exp (\alpha h),
$$

where $h$ (unit length) is the soil water potential and $\alpha$ (per unit length) is an empirical constant. The matric flux potential $\Theta$ (unit length squared per unit time) is related to the hydraulic conductivity by

$$
\Theta=\int_{-\infty}^{h} K(q) d q=\alpha^{-1} K(h) .
$$

The linearized form of the steady infiltration equation is

$$
\frac{\partial^{2} \Theta}{\partial X^{2}}+\frac{\partial^{2} \Theta}{\partial Z^{2}}=\alpha \frac{\partial \Theta}{\partial Z} .
$$

The horizontal and vertical components of the flux, as functions of the matric flux potential are

$$
U=-\frac{\partial \Theta}{\partial X},
$$




$$
V=\alpha \Theta-\frac{\partial \Theta}{\partial Z} .
$$

The flux normal to a surface with outward pointing normal $\mathbf{n}=$ $\left(n_{1}, n_{2}\right)$ is

$$
F=-\frac{\partial \Theta}{\partial X} n_{1}+\left(\alpha \Theta-\frac{\partial \Theta}{\partial Z}\right) n_{2} .
$$

Dimensionless variables are now defined in the form

$$
\begin{gathered}
\theta=\frac{1}{v_{0} L} \Theta, \quad x=\frac{\alpha}{2} X, \quad z=\frac{\alpha}{2} Z, \\
u=\frac{2}{v_{0} \alpha L} U, \quad v=\frac{2}{v_{0} \alpha L} V, \quad f=\frac{2}{v_{0} \alpha L} F,
\end{gathered}
$$

where $v_{0}$ is a reference flux. In terms of these variables equations (3) to (6) may be written in the dimensionless form

$$
\begin{gathered}
\frac{\partial^{2} \theta}{\partial x^{2}}+\frac{\partial^{2} \theta}{\partial z^{2}}=2 \frac{\partial \theta}{\partial z}, \\
u=-\frac{\partial \theta}{\partial x}, \\
v=2 \theta-\frac{\partial \theta}{\partial z} \\
f=-\frac{\partial \theta}{\partial x} n_{1}+\left(2 \theta-\frac{\partial \theta}{\partial z}\right) n_{2} .
\end{gathered}
$$

The transformation

$$
\theta=\exp (z) \Psi
$$

transforms equation (7) to

$$
\frac{\partial^{2} \Psi}{\partial x^{2}}+\frac{\partial^{2} \Psi}{\partial z^{2}}-\Psi=0 .
$$


Also equations (8-10) transform to

$$
\begin{gathered}
u=-\exp (z) \frac{\partial \Psi}{\partial x} \\
v=\exp (z)\left(\Psi-\frac{\partial \Psi}{\partial z}\right) \\
f=-\exp (z)\left[\frac{\partial \Psi}{\partial x} n_{1}-\left(\Psi-\frac{\partial \Psi}{\partial z}\right) n_{2}\right] \\
=-\exp (z)\left[\frac{\partial \Psi}{\partial n}-\Psi n_{2}\right] .
\end{gathered}
$$

Hence

$$
\frac{\partial \Psi}{\partial n}=\Psi n_{2}-e^{-z} f
$$

Due to the symmetry of the problem there will be no flow perpendicular to the vertical planes having abscissae $X=0, \pm D, \ldots$. Thus the boundary value problem need only be solved in the shaded domain shown in Figure 1 with the following boundary conditions.

The boundary condition for the soil surface outside the channels is that of no flow across the surface. Therefore from equation (14) the boundary condition on that part of the shaded region in Figure 1 that lies on $z=0$ is

$$
\Psi-\frac{\partial \Psi}{\partial z}=0
$$

Over the surface of the channel the normal flow is

$$
-\left[\frac{\partial \Psi}{\partial x} n_{1}-\left(\Psi-\frac{\partial \Psi}{\partial z}\right) n_{2}\right]=\exp (-z) f_{0}(x, z) \quad \text { for }(x, z) \in \partial \Omega_{1},
$$


where $\partial \Omega_{1}$ denotes the boundary of the channel in the shaded part of Figure 1 and $f_{0}(x, z)$ is given.

Also the condition of no flow perpendicular to the boundary lines $X=0$ and $X=-D$ of the shaded region leads to the boundary conditions

$$
\frac{\partial \theta}{\partial x}=0 \quad \text { for } \quad 0 \leq z \leq \infty \text { and } x=0 \text { and } x=-\alpha D / 2 .
$$

For $0 \geq x \geq-\alpha D / 2$ and $z=\infty[1]$

$$
\frac{\partial \theta}{\partial x}=0 \quad \text { and } \quad \frac{\partial \theta}{\partial z}=0 .
$$

Hence using (11) the corresponding conditions on $\Psi$ are

$$
\frac{\partial \Psi}{\partial x}=0 \quad \text { for } \quad 0 \leq z \leq \infty \quad \text { and } \quad x=0 \text { and } \quad x=-\alpha D / 2,
$$

and for $0 \geq x \geq-\alpha D / 2$ and $z=\infty$

$$
\frac{\partial \Psi}{\partial x}=0 \quad \text { and } \quad \frac{\partial \Psi}{\partial z}=-\Psi
$$

\section{Boundary integral equation}

The boundary integral equation for the solution to equation (12) is

$$
\lambda \Psi(a, b)=-\int_{\partial \Omega}\left[\frac{\partial \Psi}{\partial n} \phi^{\prime}-\frac{\partial \phi^{\prime}}{\partial n} \Psi\right] d S,
$$

where $\mathbf{n}=\left(n_{1}, n_{2}\right)$ is the outward pointing normal to $\Omega$ (where $\Omega$ denotes the shaded domain in Figure 1$), \lambda=1$ if $(a, b) \in \Omega$ and $\lambda=1 / 2$ if $(a, b) \in \partial \Omega$ (the boundary of $\Omega$ ) and $\partial \Omega$ has a 
continuously turning tangent. In the case of equation (12) the $\phi^{\prime}$ in equation (23) is

$$
\phi^{\prime}(x, z)=-\frac{1}{2 \pi} K_{0}^{(1)}(r) .
$$

where $r=\left((x-a)^{2}+(z-b)^{2}\right)^{1 / 2}$ and $K_{0}^{(1)}$ is a modified Bessel function.

Substitution of (16) into (23) gives

$$
\lambda \Psi(a, b)=-\int_{\partial \Omega}\left[\phi^{\prime} n_{2}-\frac{\partial \phi^{\prime}}{\partial n}\right] \Psi d S+\int_{\partial \Omega} f e^{-z} \phi^{\prime} d S .
$$

If the flux is zero across large sections of the soil surface on $z=0$ then in place of the function $\phi^{\prime}$ given by (24) it is convenient to use the Green's function

$$
\begin{aligned}
& \phi^{\prime}(x, z)=-\frac{1}{2 \pi}\left(K_{0}^{(1)}(r)+K_{0}^{(1)}(\bar{r})\right) \\
& +\frac{1}{\pi} e^{b} \int_{b}^{\infty} e^{-\mu} K_{0}^{(1)}\left(\left[(x-a)^{2}+(z+\mu)^{2}\right]^{1 / 2}\right) d \mu,
\end{aligned}
$$

where $\bar{r}=\left((x-a)^{2}+(z+b)^{2}\right)^{1 / 2}$. With this choice of the function $\phi^{\prime}$ the term $\phi^{\prime}-\partial \phi^{\prime} / \partial n$ is zero on $z=0$.

\section{$5 \quad$ Numerical results}

The boundary integral equation (25) in conjunction with the boundary conditions $(17,18,21,22)$ is used to determine the matric flux potential for a variety of configurations of the irrigation channels. In this section numerical results are given for the matric flux potential associated with infiltration from a flat strip, a semi circular channel and a rectangular channel (see Figures 2, 3 and 4). 


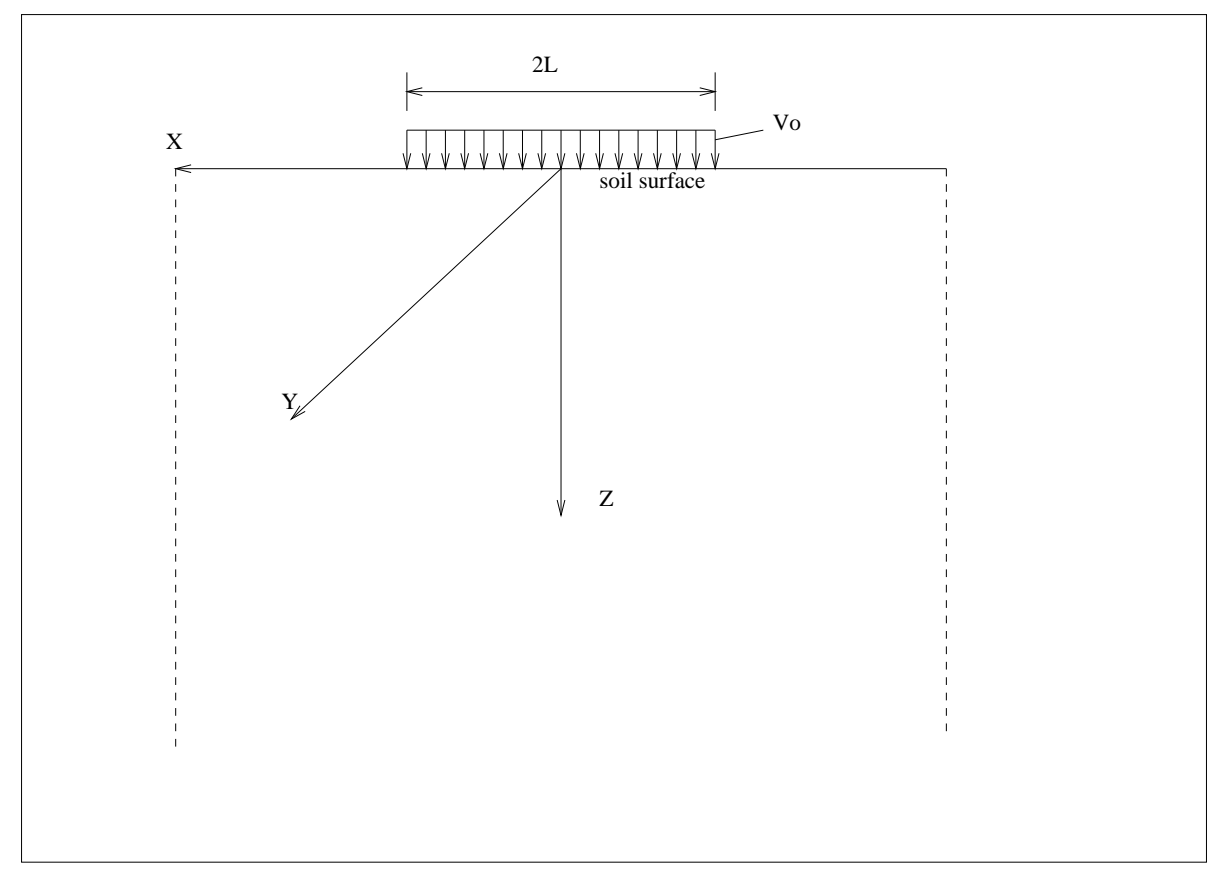

FiguRE 2: Flat strip source 


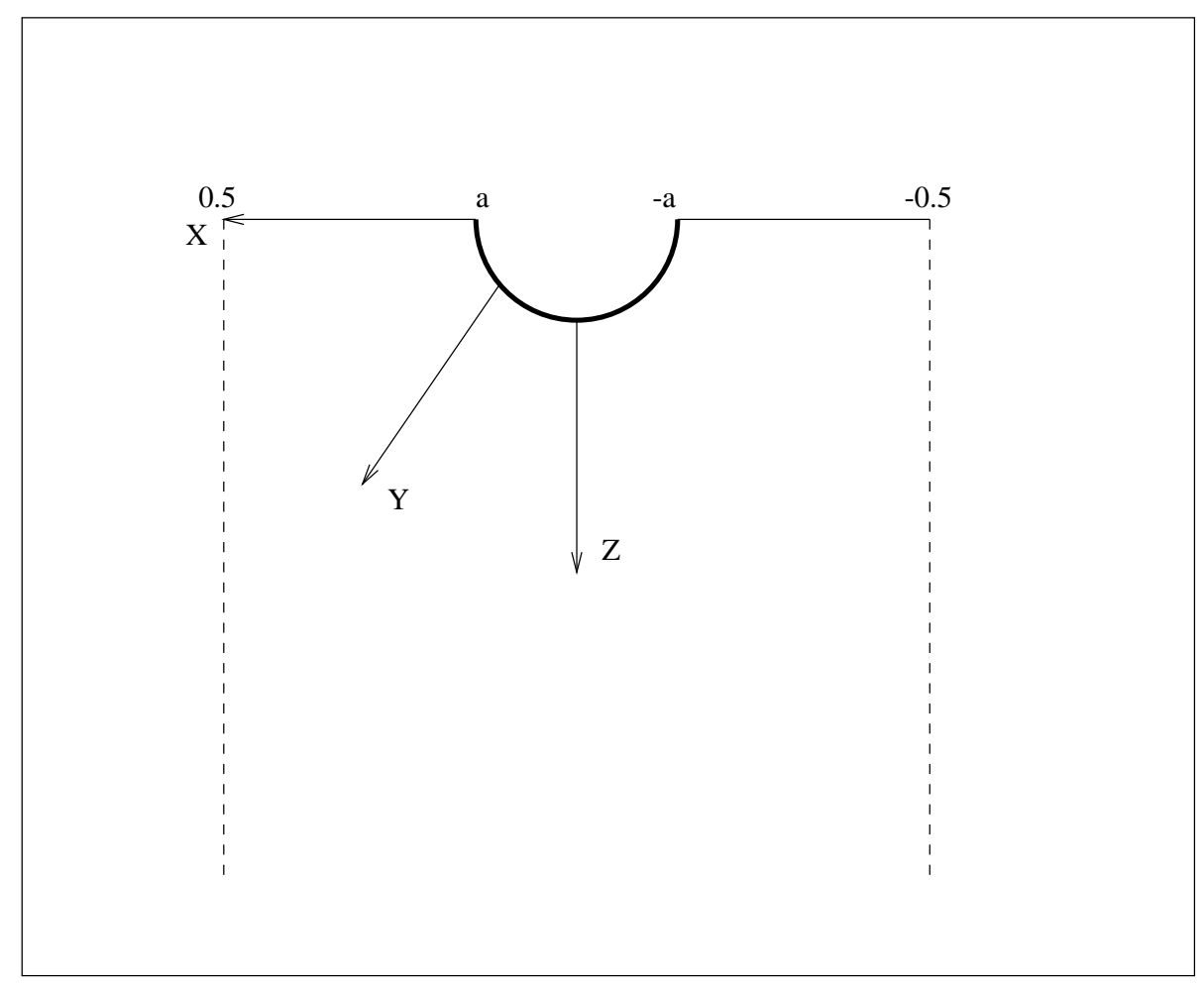

FiguRE 3: Semi-circular strip source $(a=0.2 / \pi)$ 


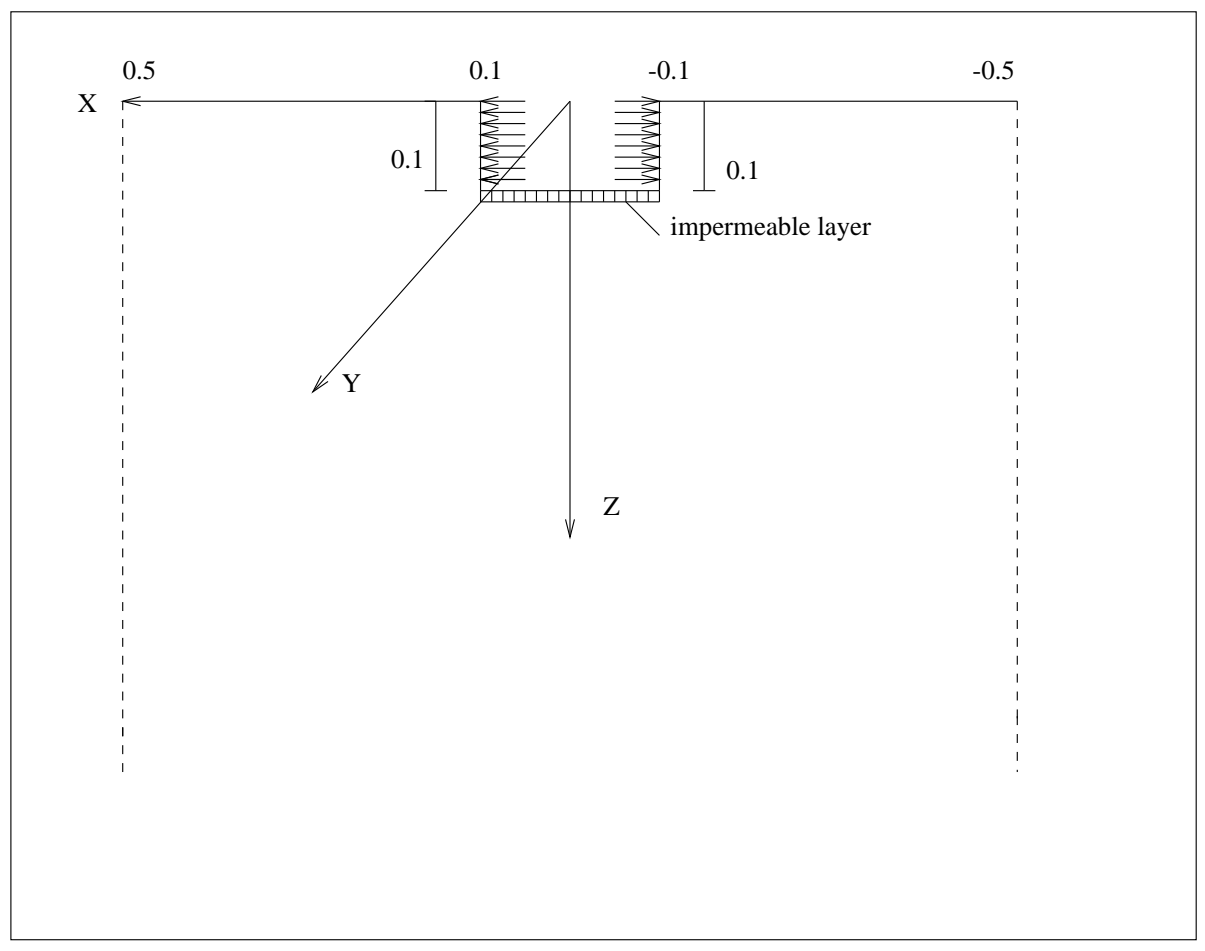

FiguRE 4: Rectangular strip source 


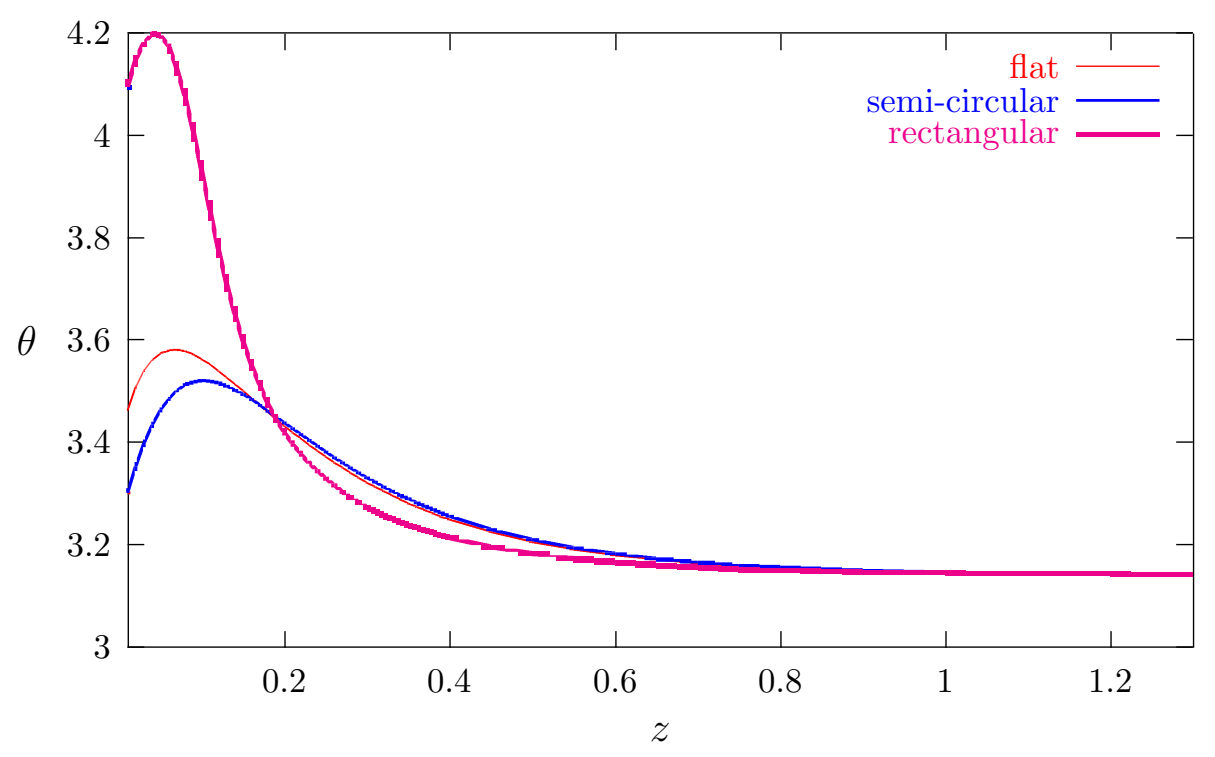

Figure 5: Values of $\theta$ along the interior line $x=0.15$

In each case numerical values of the matric flux potential on the lines $x=k$ for various values of $k$ are given in graphical form in Figures 5, 6, 7 and 8. The values of the reference length $\alpha L / 2$ and $\alpha D / 2$ are taken to be 0.1 and 0.5 respectively.

To obtain these numerical values from equation (25) standard boundary element methods were employed [3]. The boundary was divided into segments to facilitate the replacement of the integrals in (25) by a sum; thereby enabling the replacement of the integral equation (25) by a system of linear algebraic equations for the unknown function $\Psi(a, b)$. The number of segments was increased until convergence of the values of the function $\Psi(a, b)$ was achieved (to four decimal places). To obtain this level of convergence it was necessary to take the shaded region in Figure 1 to lie between the 


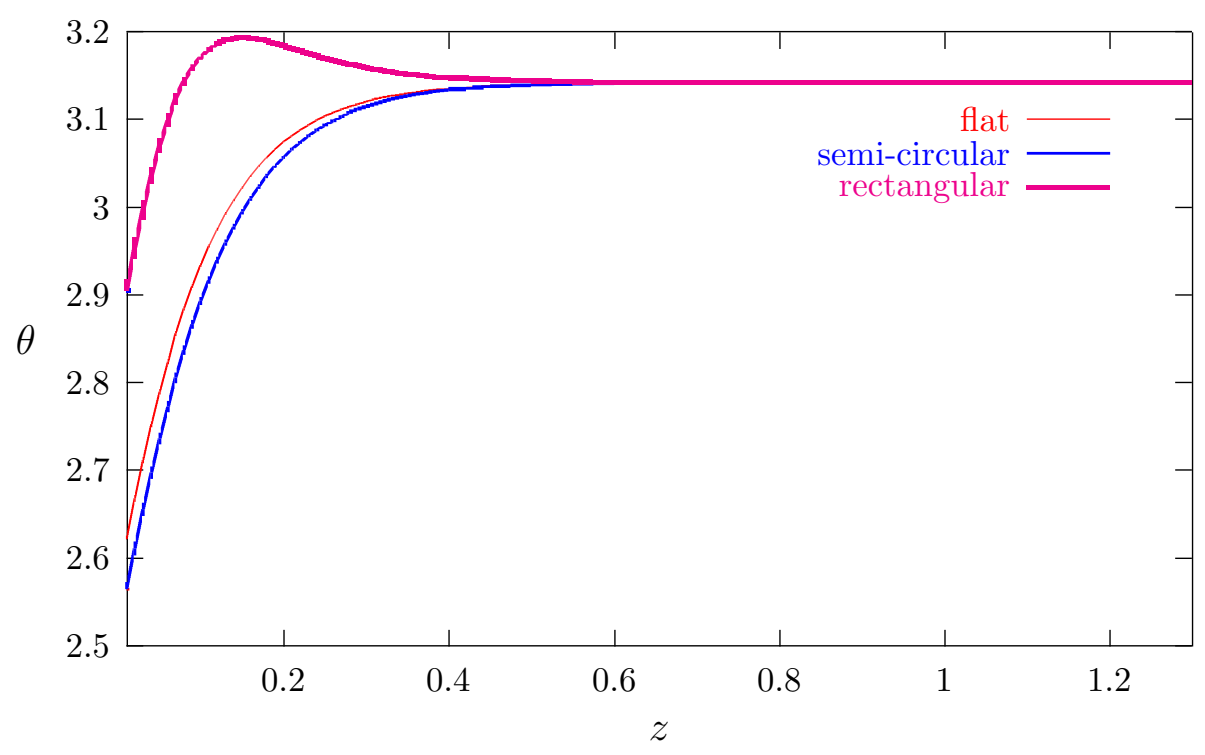

Figure 6: Values of $\theta$ along the interior line $x=0.25$ 


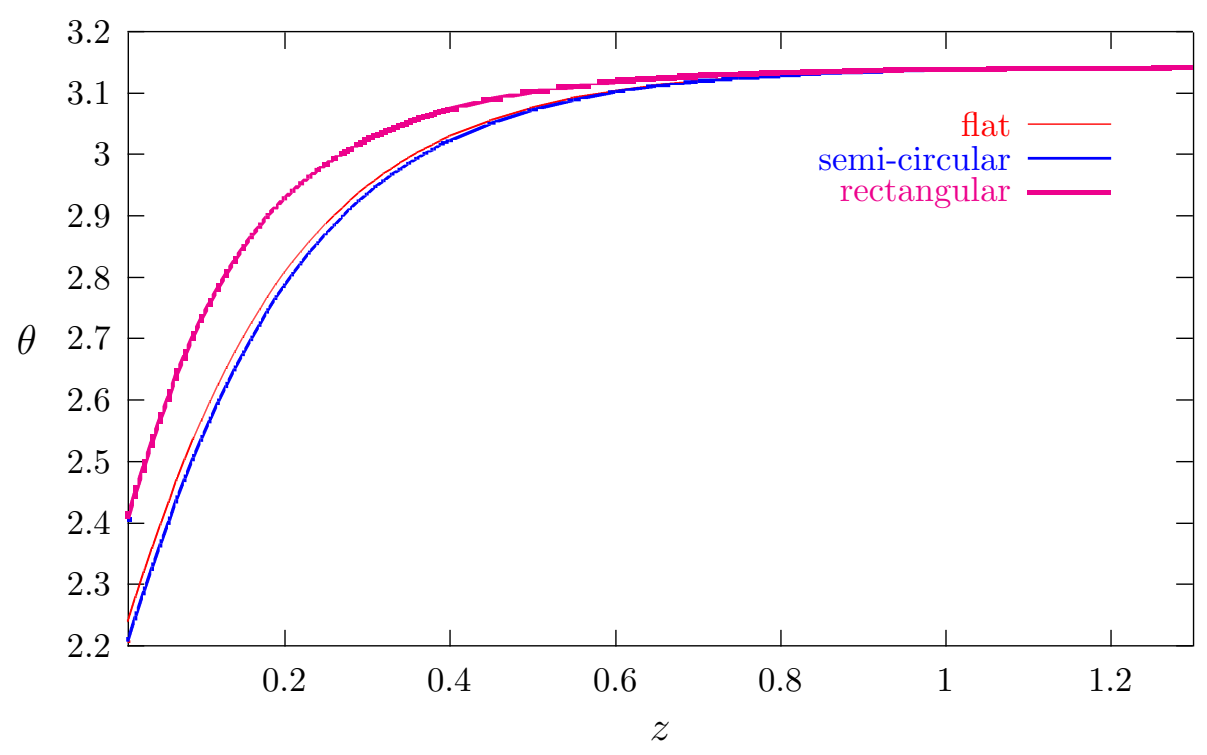

Figure 7: Values of $\theta$ along the interior line $x=0.35$ 


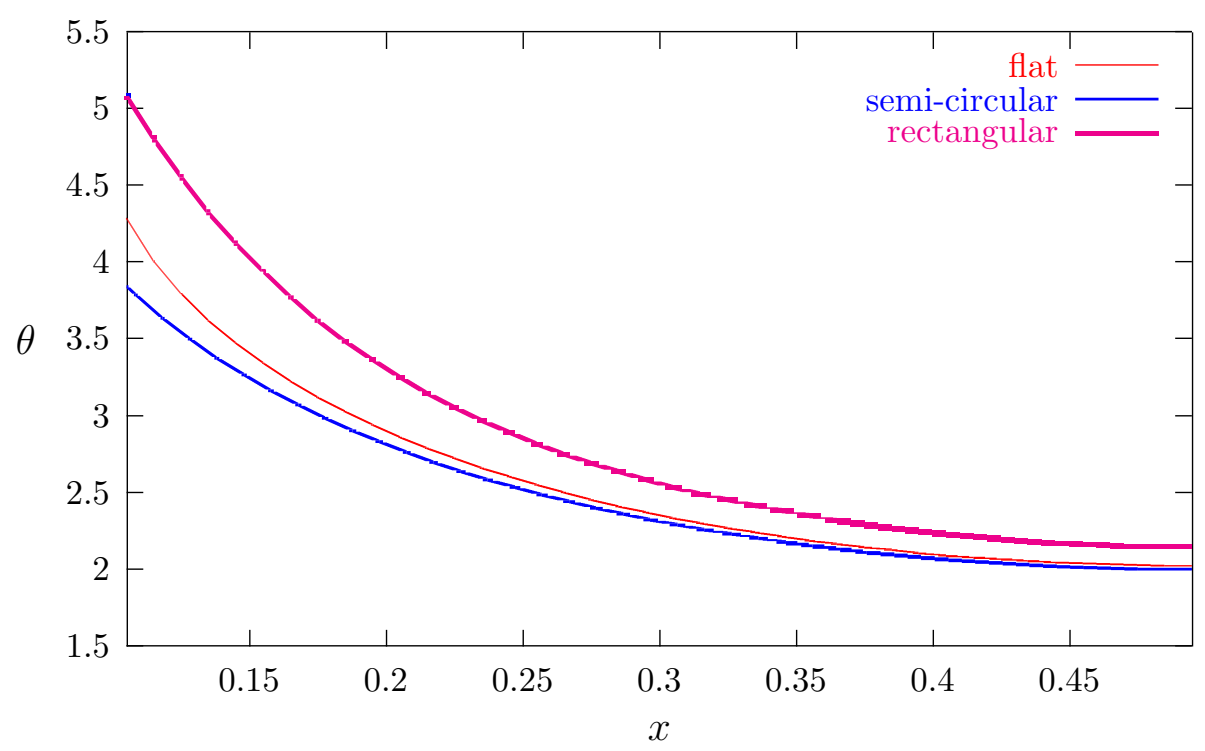

Figure 8: Values of $\theta$ along the boundary line $z=0$ 
planes $z=0$ and $z=4$ (using condition (22) $\partial \Psi / \partial z=-\Psi$ on $z=4$ ) and to divide the boundary into 1100 segments.

The numerical values shown in Figures 5-8 for the matric flux potential indicate clearly the effect of the variation of the potential for the three channel problems considered in this section. Note that in each case the surface area through which water could infiltrate was taken to be identical so that the same volume of water per unit time infiltrated from each of the three channels.

The results show that the matric flux potential is not greatly altered by replacing the flat channel by a semi-circular channel (Figure 3). In contrast replacement of the flat channel by a rectangular ditch with the bottom of the ditch covered with an impermeable layer gives substantially increased values for the matric flux potential. This is particularly the case near the surface $z=0$ where the plant roots are likely to be most evident.

\section{Summary}

A boundary element method has been obtained for the solution of a class of problems involving infiltration from a series of periodic irrigation channels. The method has been used to compare the effectiveness of some particular channel profiles by determining the distribution of the matric flux potential for the channels under consideration. The matric flux potential associated with the flat strip and semicircular channel are shown to be similar; whereas a particular rectangular channel is shown to substantially increase the potential in regions adjacent to the channel sides. 


\section{References}

[1] V. Batu. Steady Infiltration From Single And Periodic Strip Sources. Soil Science Society of America Journal, 42:545-549, 1978. C63, C64, C67

[2] V. Batu. Steady Infiltration From a Ditch : Theory and Experiment. Soil Science Society of America Journal, 41:677-682, 1977. C62

[3] D. L. Clements. Boundary Value Problems Governed By Second Order Elliptic Systems. Pitman, New York, 1990. C72

[4] D. O. Lomen and A. W. Warrick. Time Dependent Linearized Infiltration. II. Line Sources. Soil Science Society of America Proceedings, 38:568-572, 1974. C62

[5] J. R. Philip. Theory of Infiltration. Adv. Hydrosci., 5:215-296, 1971. C62

[6] J. R. Philip. General Theorem on Steady Infiltration from Surface Sources with Application to Point and Line Sources. Soil Science Society of America Proceedings, 35:867-871, 1971. C62

[7] J. R. Philip. Steady Infiltration From Burried Point Sources and Spherical Cavities. Water Resources Research, 4:1039-1047, 1968. C62

[8] P. A. C. Raats. Steady Infiltration from Point Sources, Cavities and Basins. Soil Science Society of America Proceedings, 35:689-694, 1971. C62

[9] P. A. C. Raats. Steady Infiltration from Line Sources and Furrows. Soil Science Society of America Proceedings, 34:709-714, 1970. C62 
[10] A. W. Warrick. Time-dependent Linearized Infiltration: I. Point Sources. Soil Science Society of America Proceedings, 38:383-386, 1974. C62

[11] R. A. Wooding. Steady Infiltration From Shallow Circular Pond. Water Resour. Res., 4:1259-1273, 1968. C62

[12] D. W. Zachman and A. W. Thomas. A Mathematical Investigation of Steady Infiltration from Line Sources. Soil Science Society of America Proceedings, 37:495-500, 1973. C62 